\section{Gene targeting in Drosophila}

A recent article in Science (288, 2013-2017, 2000) describes the first gene targeting technology for the fruit fly, Drosophila melanogaster. Until now, attempts at gene targeting in Drosophila have been limited to transposable $\mathrm{P}$ elements, which insert genes randomly into the genome. Authors Kent Golic and Yikang Rong accomplished specific targeting by first randomly introducing a heat-inducible site-specific I-SceI endonuclease and FLP recombinase using the $\mathrm{P}$ element system. They subsequently transformed the FLP/I-SceI flies with a targeting construct that contained a cloned copy of the gene of interest (Drosophila yellow ${ }^{+}$) flanked by FLP recombination target sites. They showed that 55 of 56 transformants detected had integrated yellow ${ }^{+}$at the target locus. Although critics suggest that the events may have been due to breakage-induced replication close to the telomere, Golic is further characterizing the system to validate the specificity of gene targeting and to increase targeting efficiency. With at least 177 genes shared between humans and fruit flies, the system should prove useful in functional genomics.

$A J B$

\section{Telomerase linked with cancer risk?}

New findings suggest that the use of telomerase to induce proliferation in cultured cells for use in therapies may be associated with malignant changes. Collaborating scientists have introduced the gene encoding human telomerase reverse transcriptase (hTERT) into human mammary epithelial cells (HMECs) and studied the effects on expression of the "oncogene" c-myc. As expected, when hTERT was introduced in cells, c-myc expression was elevated; however, when hTERT was excised, overexpression of c-myc was maintained and telomerase activity also remained high (Nature 405, 755-756, 2000). Primary investigator David Beach of the Wolfson Institute for Biomedical Research (University College, London, UK) believes that "regulation of c-myc is most likely the key to utilizing this pathway for halting cell senescence." He also maintains that there is another, earlier pathway involving the p16 tumor suppressor gene that must be studied to fully understand this system. Comparison of the p16 and c- $m y c$ regulatory pathways in cell lines other than HMECs should provide a better understanding of the role of hTERT in immortalizing cell growth.

Research briefs written by Aaron J. Bouchie, Alan Dove, and Eric Niiler.

\title{
Transgenic blood suckers
}

An international collaboration of scientists has successfully inserted green fluorescent protein (GFP) into Anopheles stephensi mosquito embryos, creating the first transgenic Anopheles mosquito and raising hopes in the fight against malaria (Nature 405, 959-962, 2000). Transformation was made possible when researchers finally were able to prevent the hardening process of the mosquito embryo, which occurs immediately after the eggs are deposited, according to senior author Andrea Crisanti. The team tested a compound that inhibited the enzyme phenoloxidase and prevented the first steps of melanization. Treated embryos stayed soft and transparent for up to $3 \mathrm{~h}$, allowing injection of the enhanced GFP gene. An average of $29 \%$ of the injected embryos survived, and half of them carried the marker gene to the larval stage. By

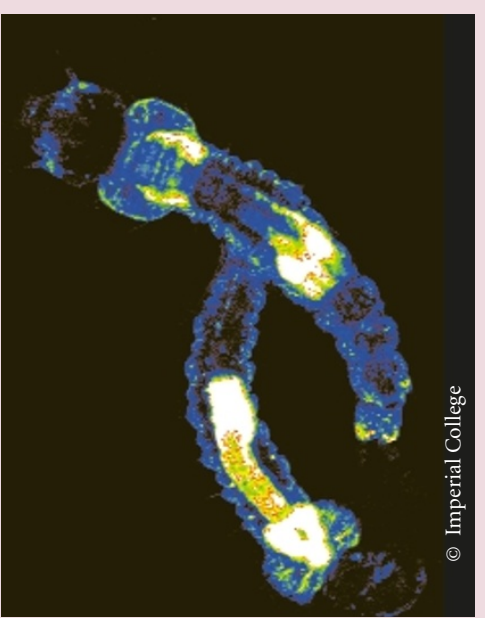
inserting the marker gene using the transposon Minos, the researchers have shown they can alter the mosquito genome. The next step is to develop a malaria-resistant mosquito by inserting genes to disrupt the interaction between the mosquito and the malaria-causing parasite, or perhaps to direct malaria-infected mosquitoes to prey on animals rather than humans, Crisanti says.

\section{Bacillus identity crisis}

Researchers at the Biotechnology Centre at the University of Oslo, led by Anne-Brit Kolstø, have determined that what were thought to be three separate bacterial species are actually three strains of the same species (Appl. Environ. Microbiol. 66, 2627-2630, 2000). This team had previously found evidence that Bacillus thuringiensis, the sire of $B t$ toxin, and B. cereus, a common cause of food poisoning found ubiquitously in the soil, appear to be the same species, exhibiting low degrees of clonality and frequent exchange of genetic material. Through multilocus enzyme electrophoresis (MEE) and sequence analysis of nine chromosomal genes, the Kolstø group now has found that B. anthracis, the cause of anthrax, belongs to the same species as well. The difference in phenotype is due to virulent plasmids harbored within $B$. anthracis. With $B$. anthracis currently undergoing complete sequencing, Kolstø plans to sequence genes in the closest $B$. cereus relatives to determine what exactly allows $B$. anthracis to retrieve and retain virulent plasmids. Although researchers should not be overly concerned by these findings, she says, they could have implications for "organic" pest control methods: "We do not know whether it would be dangerous to use $B$. thuringiensis as a whole bacterium for pesticidal reasons due to possible genetic transfer," warns Kolstø.

\section{Lentiviruses for stable gene therapy}

Researchers at the Memorial SloanKettering Cancer Center (New York, NY) and the University of California (San Francisco, CA) have used a lentiviral vector to introduce functional $\beta$-globin genes into hematopoietic stem cells, alleviating $\beta$-thalassemia in a mouse model of the disease. Although significant hurdles remain before HIV-1 and related lentiviruses can be considered for gene therapy in humans, the new work demonstrates that the vector can introduce and regulate corrected genes during stem cell development (Nature 406, $82-86,2000)$. Previous studies on $\beta$-globin gene regulation showed that the locus control region (LCR) upstream of the gene is required for properly regulated gene expression, but earlier gene therapy approaches have been unable to incorporate enough of the LCR to achieve regulated expression. Now, researchers have found that a lentiviral vector can transduce a large portion of the LCR into hematopoietic stem cells, and that the transduced gene remains stably integrated. Michel Sadelain, head of the gene transfer and gene expression laboratory at SloanKettering and senior author on the paper, says the system "could be used as a model for introducing genes in other types of stem cells as well." 